\title{
EFEITO DO TRATAMENTO TÉRMICO DA MADEIRA DE PINUS ELLIOTTII NOS PARÂMETROS FÍSICO-MECÂNICOS E COLORIMÉTRICOS
}

\author{
EFFECT OF HEAT TREAMENT OF PINUS ELLIOTII WOOD IN PHYSIC-MECHANICAL AND \\ COLORIMETRIC PARAMETERS
}
Henrique Römer Schulz ${ }^{1}$, Andrey Pereira Acosta ${ }^{2}$, Kelvin Techera Barbosa ${ }^{3}$, Ezequiel Gallio , Rafael Beltrame ${ }^{5}$, Darci Alberto Gatto ${ }^{6}$
1, 2, 3, 4, 5, 6 Universidade Federal De Pelotas, Pelotas, Rio Grande do Sul, Brasil - henriqueschulz09@hotmail.com,andreysvp@gmail.com, kelvintecherabarbosa@gmail.com, egeng.florestal@gmail.com, beltrame.rafael@yahoo.com.br \&darcigatto@yahoo.com.br

\section{RESUMO}

O objetivo deste trabalho foi avaliar o efeito do tratamento térmico nas propriedades tecnológicas da madeira de Pinus elliottii. Para tanto, utilizou-se madeira de 22 anos, de onde foram obtidos corpos de prova com dimensões de $20 \mathrm{~mm} \times 20 \mathrm{~mm} \times 50 \mathrm{~mm}$, os quais foram submetidos a tratamentos térmicos em três temperaturas distintas $\left(160^{\circ} \mathrm{C}\right.$, $200^{\circ} \mathrm{C}$ e $240^{\circ} \mathrm{C}$ ) por um período de tempo de 2 horas. Posteriormente, foram avaliadas a massa específica aparente, a perda de massa e a dureza Janka, seguindo as normas ASTM D 2395 (2017), ASTM D2017 (2005) e ASTM D143-94 (2014), respectivamente. Para a colorimetria, efetuaram-se quatro leituras em cada corpo de prova (2 no plano anatômico radial e 2 no tangencial) para obtenção dos parâmetros de claridade $\left(\mathrm{L}^{*}\right)$, matriz vermelho ( $\left.\mathrm{a}^{*}\right)$, matriz amarelo $\left(b^{*}\right)$, saturação da cor $\left(c^{*}\right)$, ângulo de tinta $\left(h^{\circ}\right)$ e variação total da cor $(\Delta E)$. Notou-se redução na massa específica aparente e perda de massa com o aumento da temperatura, justificado pela degradação dos componentes químicos de alto peso molecular. Em relação a dureza, observou-se aumento significativo para o tratamento a $160^{\circ} \mathrm{C}$. Em relação a colorimetria, notou-se redução nos parâmetros de $L^{*}, b^{*}, c^{*}$ e $h^{\circ}$, aumento nos parâmetros a* e $(\Delta E)$. Com isso, conclui-se que o tratamento térmico causa modificações nas propriedades físico-mecânicas e colorimétricas das madeiras de Pinus elliottii.

PALAVRAS-CHAVE: Colorimetria, Dureza Janka, Modificação da madeira, Retificação térmica.

\section{ABSTRACT}

The objective of this work was to evaluate the effect of heat treatment on the technological properties of Pinus elliottii wood. For this purpose, 22-year-old wood was used, from which specimens with dimensions of $20 \mathrm{~mm} \times 20 \mathrm{~mm} \times 50$ $\mathrm{mm}$ were obtained, which were subjected to heat treatment at 3 different temperatures $\left(160^{\circ} \mathrm{C}, 200^{\circ} \mathrm{C}\right)$. and $240^{\circ}$ C) for a period of 2 hours. Subsequently the apparent specific mass, mass loss and Janka hardness were evaluated following the ASTM D 2395 (2017), ASTM D2017 (2005) and ASTM D143-94 (2014) standards, respectively. (2 in the radial anatomical plane and 2 in the tangential plane) to obtain the parameters of brightness $\left(\mathrm{L}^{*}\right)$, red matrix $(\mathrm{a} *)$, yellow matrix $\left(b^{*}\right)$, color saturation $\left(c^{*}\right)$, ink angle $\left(h^{\circ}\right)$ and total color variation $(\Delta E)$. Reductions in apparent specific mass and mass loss were observed in the results with the increase in temperature justified by the degradation of the high molecular weight chemical components. Colorimetry showed a reduction in the parameters of $L^{*}, b^{*}, c^{*}$ and $\mathrm{h}^{\circ}$, an increase in the parameters $\mathrm{a}^{*}$ and $(\Delta \mathrm{E})$. Thus, it can be concluded that the heat treatment causes changes in the physical-mechanical and colorimetric properties of Pinus elliottii woods.

KEYWORDS: Colorimetry, Janka hardness, Modification of wood, Thermal grinding. 


\section{INTRODUÇÃO}

O gênero Pinus possui vantagens por ter alta produtividade, fornecendo madeira de boa qualidade, para as mais diversas finalidades. Entretanto, apresenta limitações em determinados parâmetros tecnológicos, como elevada capacidade de absorver água, além da coloração branca, necessitando em função disto de tratamentos que visem a otimização destes parâmetros em função da finalidade ao qual se destina.

Assim, almejando melhorias em determinadas propriedades, tem se realizado tratamentos térmicos, buscando modificações nos parâmetros físicos, químicos e mecânicos. Moura et al. (2012) salientam que o tratamento térmico reduz a higroscopicidade, melhorando a estabilidade dimensional, além de alterar parâmetros colorimétricos tornando a madeira com aparência mais escura semelhante à das madeiras tropicais nativas de alto valor comercial (VIITANIEMI \& THERMOWOOD, 1997; SANTOS, 2000, MOHAREB et al., 2012, MODES et al., 2017).

Neste contexto, Cademartori et al. (2012) relatam também os mínimos impactos ambientais como sendo outra vantagem dos tratamentos térmicos, pois, não utiliza produtos químicos durante o processo, expondo a madeira a diferentes temperaturas por determminados periodos de tempo (PONCSAK et al., 2011).

As alterações e intensidade dos tratamentos térmicos começam a partir de $160^{\circ} \mathrm{C}$, devido ao inicio da degradação das hemiceluloses, celuloses e alterações químicas na lignina a partir $200^{\circ} \mathrm{C}$ reduzindo o material lenhoso da madeira. Portanto, o material modificado termicamente apresenta carcateristicas distintas quando comparadas as amostras não submetidas ao tratamento térmico.

Perante o exposto, o objetivo deste estudo foi avaliar o tratamento térmico nos parâmetros tecnológicos da madeira como a perda de massa, massa específica aparente, tensão máxima de Dureza Janka do plano transversal e análise colorimétrica da madeira de Pinus elliottii, em distintas temperaturas por um mesmo período de tempo.

\section{MATERIAL E MÉTODOS}

No presente estudo foi utilizada madeira de Pinus elliottii com 22 anos de idade. Os corpos de prova foram confeccionados com dimensões de $20 \mathrm{~mm} \times 20 \mathrm{~mm} \times 50$ $\mathrm{mm}$ (tangencial, radial e longitudinal). As amostras foram separadas em quatro grupos sendo 3 tratamentos térmicos $\left(\mathrm{T} 160^{\circ} \mathrm{C}-160^{\circ} \mathrm{C}, \mathrm{T} 200^{\circ} \mathrm{C}-200^{\circ} \mathrm{C}\right.$ e $\mathrm{T} 240^{\circ} \mathrm{C}-240^{\circ} \mathrm{C}$ ), por um período de 2 horas, em uma estufa laboratorial (sem circulação de ar) e um tratamento controle. Posteriormente a cada tratamento, os corpos de prova foram dispostos em câmara climatizada $\left(20^{\circ} \mathrm{C}\right.$ e $65 \%$ U.R.) até atingirem o equilíbrio higroscópico, para assim realizarem-se os ensaios das propriedades físicas, propriedades mecânicas, e análise colorimétrica.

A massa específica aparente foi determinada utilizando a equação 1 , de acordo com a recomendação da norma da American Society for Testing and Materials - ASTM D 2395 (2017), com auxílio de um paquímetro digital e balança analítica.

$$
\rho_{12 \%}=\left(\frac{\mathrm{M}_{12 \%}}{\mathrm{~V}_{12 \%}}\right)
$$

Em que: $\mathrm{M}_{12 \%}=$ massa a $12 \%$ de teor de umidade (g); $\mathrm{V}_{12 \%}=$ volume com a amostra a $12 \%$ de teor de umidade $\left(\mathrm{cm}^{3}\right)$; e $\rho_{12}=$ massa específica aparente a $12 \%\left(\mathrm{~g} / \mathrm{cm}^{3}\right)$.

A determinação da perda de massa foi efetuada por meio da normatização ASTM D2017 (2005), a partir da variação entre os valores de massa antes e após os tratamentos térmicos, conforme a equação 2 .

$$
\mathrm{PM}_{\%}=\frac{\mathrm{M}_{\mathrm{i}}-\mathrm{M}_{\mathrm{f}}}{\mathrm{M}_{\mathrm{i}}}
$$

Em que: $\mathrm{PM} \%=$ perda de massa (\%); $\mathrm{M}_{\mathrm{i}}=$ massa dos corpos de prova em equilíbrio a $12 \%$ (g); e $\mathrm{M}_{\mathrm{f}}=$ massa dos corpos de prova depois da termorretificação (g).

Nas propriedades mecânicas foram consideradas a Dureza Janka, a qual foi determinada utilizando máquina universal de ensaios EMIC, com capacidade de carga de $300 \mathrm{kN}$, sendo baseado em técnicas normativas da ASTM D143-94 (2014), verificando-se o esforço necessário para introduzir uma semiesfera de aço com 1,0 cm de diâmetro, sendo feito uma leitura na face transversal de cada amostra.

Para as análises colorimétricas, efetuaram-se quatro leituras para cada corpo de prova (duas na superfície radial e duas na tangencial), utilizando um colorímetro Konica Minolta, modelo CR-400, com fonte iluminante D65 e ângulo de observação de $10^{\circ}$.

O aparelho forneceu os parâmetros e claridade $\left(L^{*}\right)$, coordenada vermelho-verde ou matriz vermelho $\left(a^{*}\right)$, coordenada azul-amarelo ou matriz amarelo $\left(b^{*}\right)$, saturação da cor ( $\left.c^{*}\right)$ e o ângulo de tinta $\left(h^{*}\right)$, baseados no sistema de cor CIEL $a^{*} b^{*}$ 1976. Para determinar as alterações de cor, foi utilizada a Equação (3).

$$
\Delta \mathrm{E}=\sqrt{\Delta \mathrm{L}^{2}+\Delta \mathrm{a}^{2}+\Delta \mathrm{b}^{2}}
$$

Em que: $\Delta \mathrm{E}=$ variação total da cor das madeiras; $\Delta \mathrm{L}=$ 
variação do gradiente de claridade; $\Delta a=$ variação do gradiente da coordenada vermelho-verde; e $\Delta b$ = variação do gradiente da coordenada azul-amarelo.

A análise estatística dos valores das propriedades tecnológicas foi realizada através de análise de variância simples (ANOVA) e quando constatada a diferença significativa entre os tratamentos fez-se a comparação de médias através do teste LSD de Fisher, com 5\% de probabilidade de erro, seguindo-se a realização da correlação de Pearson.

\section{RESULTADOS E DISCUSSÃO}

Em relação aos tratamentos térmicos aplicados na madeira de Pinus elliotti, pode-se verificar na tabela 1 que a massa específica aparente (12\%) não apresentou diferença significativa estatisticamente). Conforme Hill (2006), isso ocorre em função da relação da perda de massa e do volume após os tratamentos. Desse modo, em tratamentos que a variação de massa e do volume acontece de maneira semelhante, não acontecerá alteração da massa específica básica da madeira.

Os resultados para a massa específica apresentaram a mesma tendência do estudo de Conte et al. (2014), onde analisaram diferentes tratamento de termorretificação para a madeira de Pinus elliottii com 20 anos de idade, não apresentando diferença para essa propriedade.

Segundo Calonego et al. (2012), esse resultado indica que, durante a termorretificação, o aumento de temperatura não provocou nas amostras uma diminuição de massa proporcional à diminuição de volume, apontando que a massa específica básica não se apresenta como um parâmetro para avaliar a qualidade da termorretificação.

Para a perda da massa, verificou-se um comportamento distinto entre os tratamentos de termorretificação, sendo que o aumento da temperatura causou perda de massa mais acentuada nas amostras. 0 tratamento com temperatura de $240^{\circ} \mathrm{C}$ ocasionou a maior perda de massa.

A perda de massa da madeira após a termorretificação está relacionada com a decomposição dos constituintes químicos, principalmente as hemiceluloses (HILL, 2006). Para Aydemir et al. (2011), temperaturas acima de $140^{\circ} \mathrm{C}$ interferem na decomposição das hemiceluloses, sendo pronunciado acima de $180^{\circ} \mathrm{C}$.

Tabela 1. Estatística descritiva para massa específica aparente a $12 \%$ ( $\rho_{12} \%$ ) e perda de massa (PM) e tensão máxima de Dureza Janka do plano transversal ( $\sigma$ Máx) para a madeira de Pinus elliottii.

\begin{tabular}{|c|c|c|c|c|c|c|}
\hline \multirow[t]{2}{*}{ Trat } & \multicolumn{2}{|c|}{ 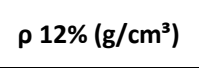 } & \multicolumn{2}{|c|}{ PM (\%) } & \multicolumn{2}{|c|}{$\begin{array}{c}\sigma \sigma_{\text {Máx }} \text { Transversal } \\
(\mathrm{MPa})\end{array}$} \\
\hline & Média & CV (\%) & Média & CV (\%) & Média & CV (\%) \\
\hline Cont & $\begin{array}{c}0,509 \\
\text { A }\end{array}$ & 5,4 & - & - & $\begin{array}{c}33,66 \\
\text { A }\end{array}$ & 23,2 \\
\hline $\mathrm{T}_{160^{\circ} \mathrm{C}}$ & $\begin{array}{c}0,495 \\
A\end{array}$ & 4,5 & $\begin{array}{c}3,47 \\
\mathrm{~A}\end{array}$ & 2,8 & $\begin{array}{c}37,36 \\
B C\end{array}$ & 12,3 \\
\hline $\mathrm{T}_{200^{\circ} \mathrm{C}}$ & $\begin{array}{c}0,487 \\
\text { A }\end{array}$ & 9,4 & $\begin{array}{c}7,38 \\
\text { B }\end{array}$ & 24,6 & $\begin{array}{c}41,94 \\
\text { C }\end{array}$ & 4,1 \\
\hline $\mathrm{T}_{240^{\circ} \mathrm{C}}$ & $\begin{array}{c}0,476 \\
\text { A }\end{array}$ & 7,0 & $\begin{array}{c}13,98 \\
C\end{array}$ & 6,1 & $\begin{array}{c}31,82 \\
A B\end{array}$ & 12,4 \\
\hline Teste $\mathrm{F}$ & 0,98 & $\begin{array}{l}\text { TCV } \\
(6,7)\end{array}$ & $\begin{array}{c}237,03 \\
*\end{array}$ & $\begin{array}{l}\text { TCV } \\
(86)\end{array}$ & $\begin{array}{c}6,9 \\
*\end{array}$ & $\begin{array}{l}\text { TCV } \\
(18)\end{array}$ \\
\hline
\end{tabular}

Em que: Trat = tratamentos; Cont $=$ controle; $\rho_{12 \%}=$ Massa específica aparente a $12 \%$; $\sigma$ Máx Transversal = tensão máxima na face transversal; Valores entre parênteses = desvio padrão na unidade da medida de cada propriedade; Letras diferentes, nas colunas, indicam diferenças significativas entre as espécies, conforme o teste LSD Fisher, em 95\% de confiança; CV = coeficiente de variação (\%); TCV = coeficiente de variação total; $\mathrm{e}^{*}$ - significativo em $5 \%$ de probabilidade de erro, pelo teste $F(p<0,05)$.

Conforme Esteves et al. (2007), em seu estudo com a espécie de Eucalyptus globulus, a perda de massa após o processo de termorretificação está ligada a baixa estabilidade térmica das hemiceluloses, que são degradadas durante os tratamentos. Conte et al. (2014), estudando a diferentes tratamentos de termorretificação $\left(130\right.$ a $210^{\circ} \mathrm{C}$ ) para a madeira de Pinus elliottii com 20 anos de idade, obtiveram uma variação de perda massa de $0,7 \%$ a $6,67 \%$, havendo tendência de crescimento conforme o aumento da temperatura.

Quando comparado o presente estudo, verifica-se que uma perda de massa mais acentuada, podendo estar relacionada com a temperatura final utilizada no estudo $\left(240^{\circ} \mathrm{C}\right)$.

Para a tensão máxima do teste de dureza Janka, observa na Tabela 1 diferenças estatísticas entre os tratamentos. Porém, os resultados obtidos não apresentaram tendência normal a resistência a dureza em relação ao aumento da temperatura. Sendo que o tratamento a $200^{\circ} \mathrm{C}$ apresentou a maior resistência a dureza.

Para Boonstra et al. (2007), o tratamento térmico pode contribuir positivamente nas propriedades mecânicas pelo fato de que a madeira tratada termicamente tem a capacidade reduzida de trocar água com o meio e, assim, o baixo conteúdo de umidade torna a madeira mais resistente a esforços mecânicos.

Segundo o estudo de Ates et al. (2009), após tratarem a madeira de Pinus brutia Ten. a $180^{\circ} \mathrm{C}$ por $2 \mathrm{~h}$, observaram 
aumento da dureza de topo em $7 \%$ em relação as amostras controle (madeira em condições normais). Já no presente estudo, apenas o tratamento com temperatura de $240^{\circ} \mathrm{C}$ não apresentou valores maiores de resistência a dureza quando comparado com a amostra controle, pois houve uma elevada degradação térmica.

Acerca do tratamento de termorretificação em diferentes faixas de temperatura aplicado na madeira de Pinus elliottii, observou-se que todas as variáveis colorimétricas analisadas apresentaram variações quando comparados aos tratamentos controle, independentemente do plano anatômico considerado.

Essa variação de tonalidade é comprovada quantitativamente pela redução significativa e gradativa na luminosidade $-L^{*}$ (Figura 1 ) em função do aumento das temperaturas de tratamento térmico: $160^{\circ} \mathrm{C}(15,89 \%$ tangencial; $8,59 \%$ - radial), $200^{\circ} \mathrm{C}(41,11 \%$ - tangencial; $33,21 \%$ - radial $)$ e $240^{\circ} \mathrm{C}(64,40 \%$ - tangencial; $58,89 \%$ radial).

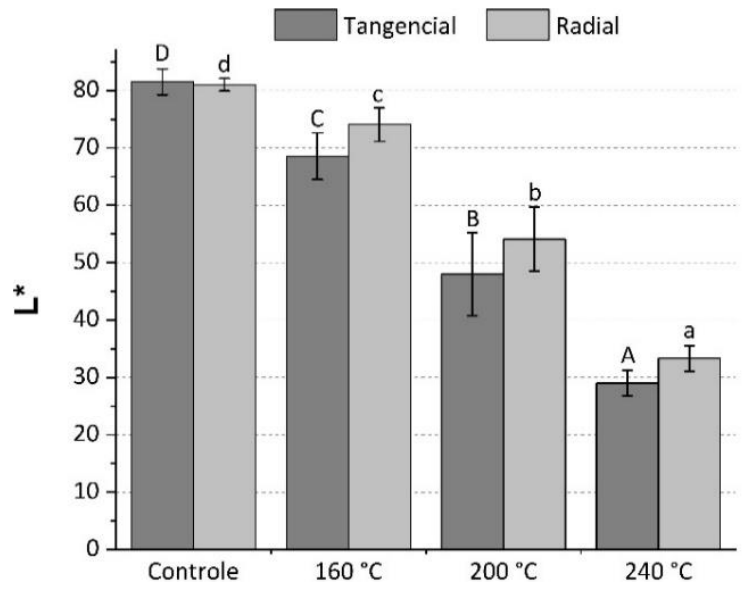

Figura 1. Variação de luminosidade $\left(L^{*}\right)$ da madeira de $P$. elliottii termorretificada em diferentes faixas de temperatura.

Em que: as linhas verticais apresentam o desvio padrão da média e letras iguais, maiúsculas no plano tangencial e minúsculas no plano radial, não apresentam diferença significativa em $5 \%$ de probabilidade de erro $(p<0,05)$ pelos testes $F$ e de Tukey.

A redução nos valores dessa variável comprova o escurecimento da madeira, uma vez que varia de 0 a 100, correspondendo as cores branca e preta, respectivamente (PINCELLI et al., 2012; ZANUNCIO et al., 2014). O escurecimento da madeira em função do tratamento térmico também foi observado em outros estudos, tais como aqueles desenvolvidos por Pincelli et al. (2012), Cademartori et al. (2013) e Zanuncio et al. (2014), os quais submeteram madeiras de Pinus caribaea (20 anos), Eucalyptus grandis (17 anos) e Eucalyptus grandis (15 anos), respectivamente, notaram a ocorrência do escurecimento da madeira em função da temperatura e do tempo de tratamento aplicados.

Acerca da matriz verde-vermelho $\left(a^{*}\right)$, os tratamentos de termorretificação aumentaram significativamente os seus valores tanto para o plano radial $(26,26 \%$ - $160 \mathrm{C}$; $141,21 \%-200^{\circ} \mathrm{C} ; 51,52 \%-240^{\circ} \mathrm{C}$ ), quanto para o tangencial $\left(72,17 \%-160^{\circ} \mathrm{C} ; 188,92 \%-200^{\circ} \mathrm{C} ; 50,71 \%-240^{\circ} \mathrm{C}\right)$, quando comparados com as amostras controle (Figura 2).

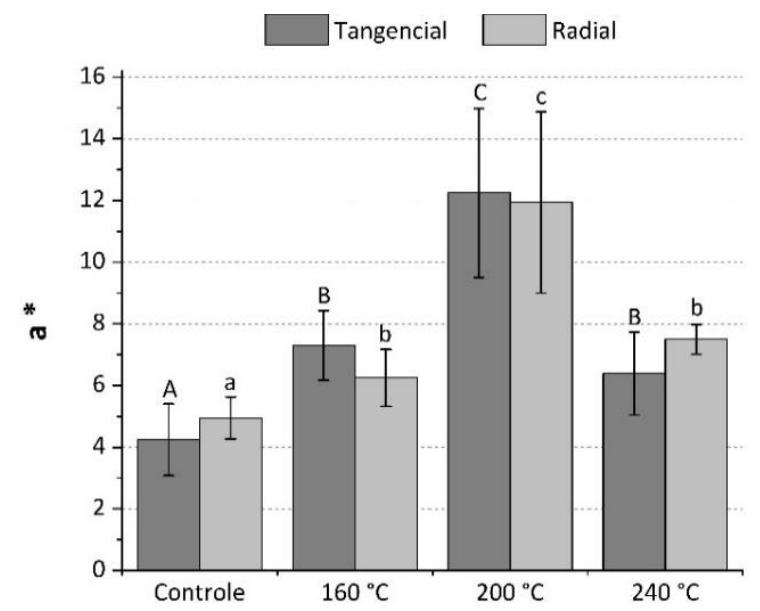

Figura 2. Variação da coordenada cromática verdevermelho $\left(a^{*}\right)$ da madeira de $P$. elliottii termorretificada em diferentes faixas de temperatura.

Em que: as linhas verticais apresentam o desvio padrão da média e letras iguais, maiúsculas no plano tangencial e minúsculas no plano radial, não apresentam diferença significativa em $5 \%$ de probabilidade de erro $(p<0,05)$ pelos testes F e de Tukey.

Os aumentos nos valores dessa variável indicam que a madeira está adquirindo uma tonalidade mais avermelhada, pois enquanto valores positivos indicam tonalidade vermelha, os valores negativos tendem ao verde (ESTEVES et al., 2008; PINCELLI et al., 2012).

Já considerando o aumento e posterior redução dos valores dessa variável $\left(a^{*}\right)$ em função do aumento das temperaturas de tratamento térmico, tal variação está associada principalmente aos extrativos, devido ao processo de volatilização e posterior degradação dos mesmos (MOURA; BRITO, 2011; CHEN et al., 2012).

A coordenada cromática ou matriz azul-amarelo ( $\left.b^{*}\right)$, tanto no tangencial quanto no radial, inicialmente apresentou tendência de acréscimo nas temperaturas de $160^{\circ} \mathrm{C}\left(14,27 \%\right.$ no tangencial e $6,67 \%$ no radial) e $200^{\circ} \mathrm{C}$ $(2,91 \%$ no tangencial e $13,62 \%$ no radial), reduzindo significativamente após tratamento térmico à $240^{\circ} \mathrm{C}$ (60,83\% no tangencial e $51,98 \%$ no radial), quando comparada ao tratamento controle (Figura 3 ). 


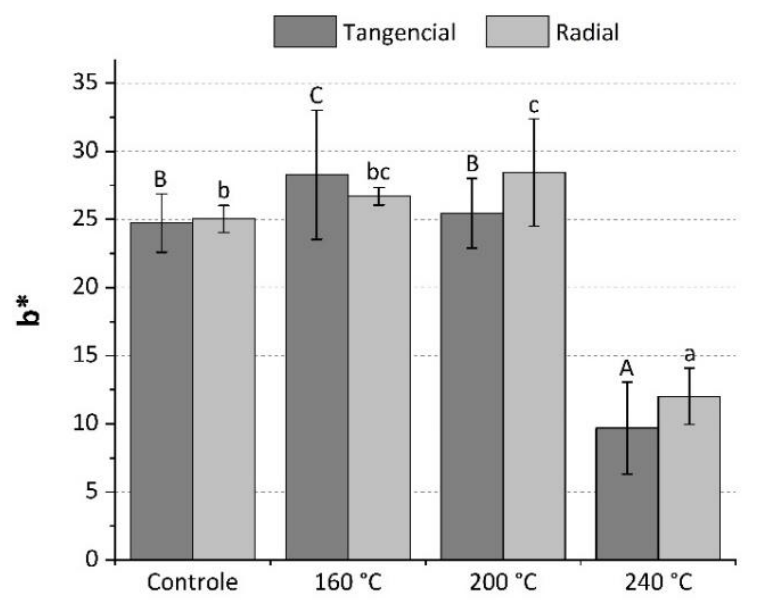

Figura 3. Variação da coordenada cromática azul-amarelo $\left(b^{*}\right)$ da madeira de $P$. elliottii termorretificada em diferentes faixas de temperatura.

Em que: as linhas verticais apresentam o desvio padrão da média e letras iguais, maiúsculas no plano tangencial e minúsculas no plano radial, não apresentam diferença significativa em $5 \%$ de probabilidade de erro $(p<0,05)$ pelos testes $F$ e de Tukey.

As reduções nos valores dessa variável indicam que a madeira está escurecendo, adquirindo uma tonalidade azulada devido a degradação de alguns constituintes químicos da madeira, como a lignina os polifenóis, bem como, compostos cromóforos associados as ligninas e aos extrativos que conferem cor amarela (ESTEVES et al., 2008; PINCELLI et al., 2012).

Nesse contexto, o perfil de variação colorimétrica da saturação - C* (Figura 4) foi semelhante ao observado na matriz $b^{*}$, descrita acima. Isso deve-se a predominância da matriz $b^{*}$ (maiores valores), em comparação com a matriz a*.

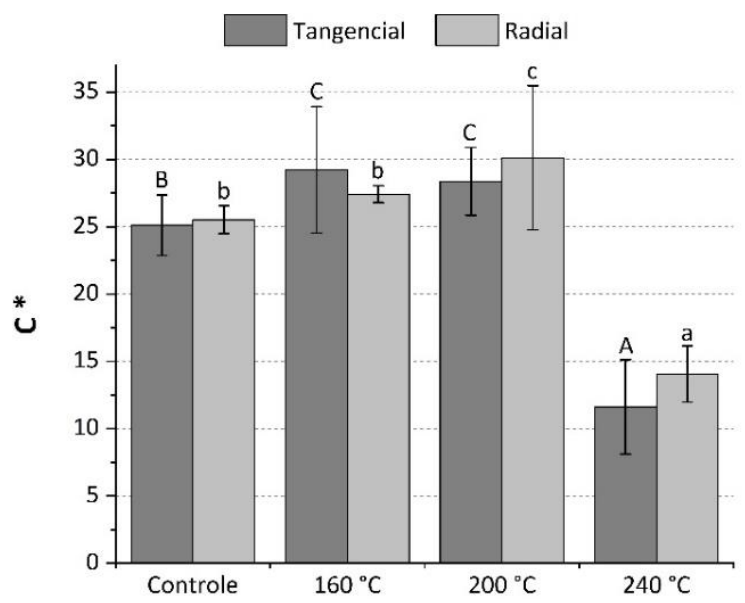

Figura 4. Variação da saturação $\left(C^{*}\right)$ da madeira de $P$. elliottii termorretificada em diferentes faixas de temperatura.

Em que: as linhas verticais apresentam o desvio padrão da média e letras iguais, maiúsculas no plano tangencial e minúsculas no plano radial, não apresentam diferença significativa em $5 \%$ de probabilidade de erro $(p<0,05)$ pelos testes $F$ e de Tukey.
Observam-se diferenças significativas principalmente à $240{ }^{\circ} \mathrm{C}$, com redução de aproximadamente $53,74 \%$ no plano tangencial e $44,87 \%$ no radial. Considerando que essa variável $\left(C^{*}\right)$ indica a pureza da cor em relação ao branco (CADEMARTORI et al., 2013), sua redução em função do tratamento térmico propõe que a madeira está tendendo à uma tonalidade acinzentada (cor menos pura).

Quanto ao ângulo de tinta $\left(\mathrm{h}^{\circ}\right)$, as temperaturas aplicadas acarretaram em reduções significativas e gradativas nos valores dessa variável a medida que a temperatura se elevava (Figura 5), em ambos os planos anatômicos: tangencial $\left(160{ }^{\circ} \mathrm{C}-6,11 \% ; 200{ }^{\circ} \mathrm{C}-19,97 \%\right.$; $\left.240{ }^{\circ} \mathrm{C}-31,06 \%\right)$ e radial $\left(160{ }^{\circ} \mathrm{C}-2,28 \% ; 200{ }^{\circ} \mathrm{C}-14,61 \%\right.$; $\left.240{ }^{\circ} \mathrm{C}-25,79 \%\right)$.

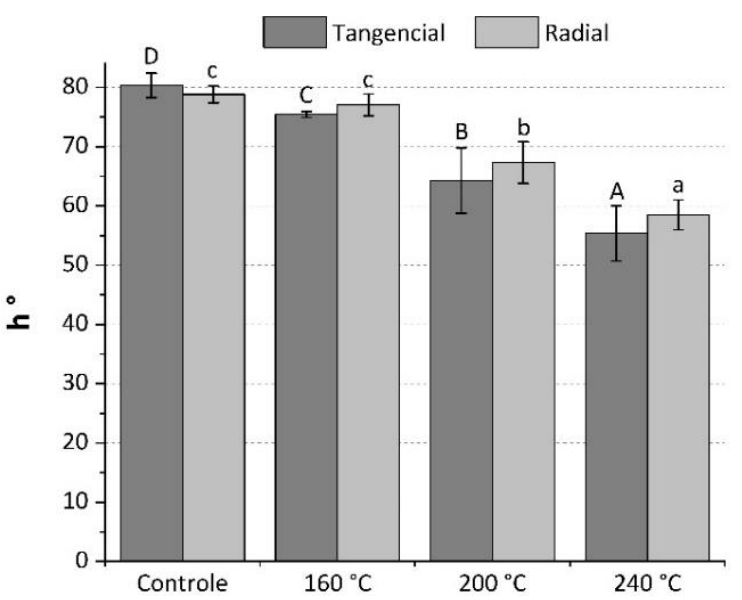

Figura 5. Variação do ângulo de tinta $\left(\mathrm{h}^{\circ}\right)$ da madeira de $P$. elliottii termorretificada em diferentes faixas de temperatura.

Em que: as linhas verticais apresentam o desvio padrão da média e letras iguais, maiúsculas no plano tangencial e minúsculas no plano radial, não apresentam diferença significativa em $5 \%$ de probabilidade de erro $(p<0,05)$ pelos testes $F$ e de Tukey.

Considerando que o $h^{\circ}$ associa-se a predominância de uma determinada tonalidade (ZANUNCIO et al., 2014), principalmente aquela relacionada à coordenada cromática predominante $\left(b^{*}\right)$. Entretanto, observa-se que em função do tratamento térmico à $240{ }^{\circ} \mathrm{C}$ houve tendência de inversão de matrizes ( $b^{*}$ diminui e $a^{*}$ aumenta, quando comparado ao controle), comprovando o escurecimento da madeira, pois a mesma parte de uma tonalidade mais amarelada $\left(b^{*}\right)$ para o vermelho $\left(a^{*}\right)$.

Percebe-se um aumento progressivo na variação total da $\operatorname{cor}(\Delta \mathrm{E})$ em função da elevação da temperatura do tratamento térmico (Figura 6). Nota-se que o plano radial apresentou menor $\Delta \mathrm{E}$ quando comparado ao tangencial. 


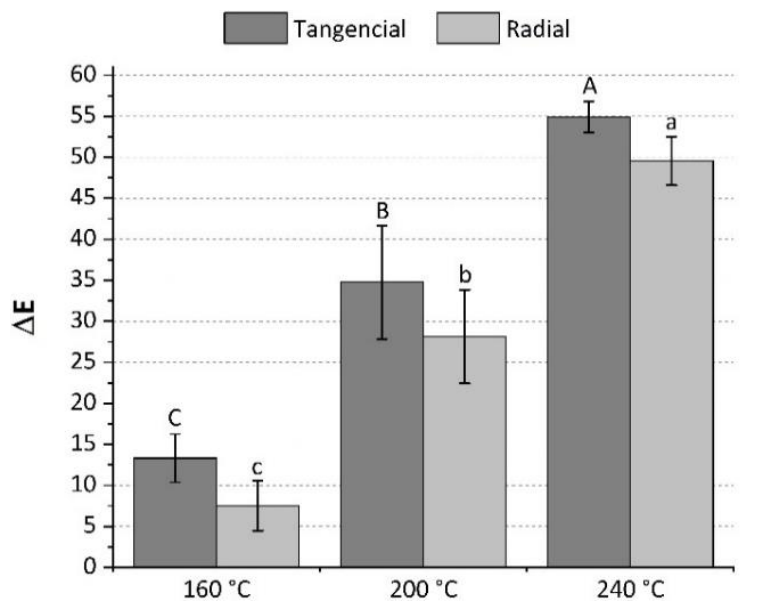

Figura 6. Variação total da $\operatorname{cor}(\Delta \mathrm{E})$ da madeira de $P$. elliottii termorretificada em diferentes faixas de temperatura.

Em que: as linhas verticais apresentam o desvio padrão da média e letras iguais, maiúsculas no plano tangencial e minúsculas no plano radial, não apresentam diferença significativa em 5\% de probabilidade de erro $(p<0,05)$ pelos testes $F$ e de Tukey.

Tendo em vista a capacidade e consequente limitação da distinção de cores pelo olho humano, o $\Delta \mathrm{E}$ é capaz de distinguir cores de madeira quando for superior à 2 (SUNDEQVIST \& MÓREN, 2002). Ficando melhor representado nas variações de cores em função dos tratamentos e entre os diferentes planos anatômicos dos corpos de prova de Pinus elliottii (Figura 7).

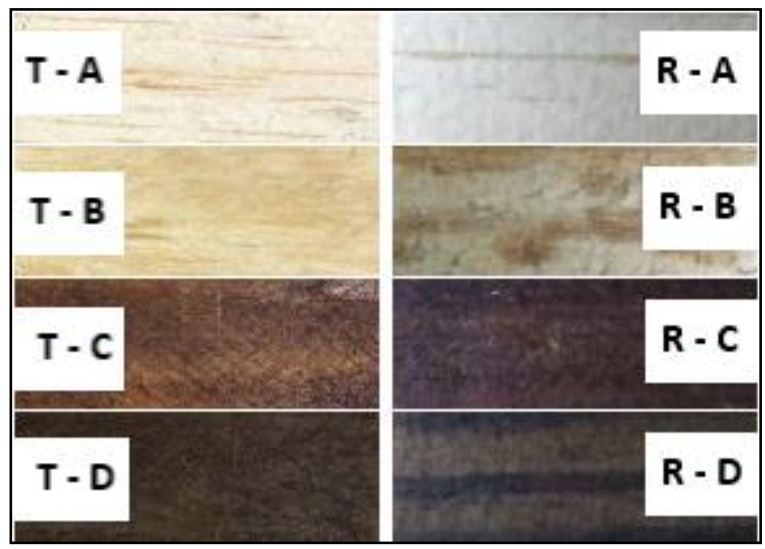

Figura 7. Variação colorimétrica dos planos anatômicos tangencial (T) e radial (R) da madeira de $P$. elliottii do grupo controle $(A)$ e termorretificada em diferentes faixas de temperatura $\left(B-160^{\circ} \mathrm{C}\right.$, $\mathrm{C}-200^{\circ} \mathrm{C}$ e D $-240^{\circ} \mathrm{C}$ ).

Em um contexto geral, o plano anatômico radial apresentou tonalidade mais escura quando comparado ao tangencial. Essa diferença de cores está associada a diferença de orientações dos elementos nos diferentes planos anatômicos da madeira (NISHINO et al., 2000).
Todos estes argumentos são corroborados pela relação de dependência entre as variáveis colorimétricas com as propriedades físicas e mecânicas avaliadas no estudo (Tabela 2). É relevante destacar a correlação elevada, direta e significativa existente entre uma mesma variável colorimétrica em um plano anatômico distinto (coeficientes superiores à 0,8 ).

Diante disso, nota-se que $\rho 12 \%$ correlacionou-se significativamente e diretamente com a $L^{*}$ e $h^{\circ}$, e inversamente com a matriz a*, para ambos os planos anatômicos (radial e tangencial).

A tensão máxima ( $\sigma_{\text {Máx }}$ ) correlacionou-se significativamente com a matriz verde-vermelha $\left(a^{*} R\right)$ e com a saturação no plano anatômico radial $\left(C^{*} R\right)$. Considerando as luminosidades tangencial $\left(L^{*} T\right)$ e radial $\left(L^{*} R\right)$, não foi observada correlação significativa somente para $L^{*} T$ com a $C^{*} R$. Ainda de acordo com a $L^{*}$, percebe-se a forte correlação entre os dois planos anatômicos, bem como a relação de dependência com a ângulo de tinta $\left(h^{\circ}\right)$.

Nota-se que as coordenadas cromáticas $a^{*}$ e $b^{*}$ independem entre si, apesar de ambas possuírem forte influência significativa na saturação $\left(C^{*}\right)$ e no ângulo de tinta $\left(h^{\circ}\right)$, principalmente a matriz $b^{*}$, comprovando sua predominância (maiores valores). Por fim, a $C^{*}$ em ambos os planos anatômicos apresenta correlação direta e significativa com o $\mathrm{h}^{\circ}$.

\section{CONCLUSÕES}

Ao se analisar o processo de retificação térmica, constata-se modificação nas propriedades físicomecânicas e colorimétricas analisadas. A massa específica aparente apresentou redução não significativa à medida que houve aumento da temperatura de tratamento.

A perda de massa reduziu com aumento da temperatura, sendo que no tratamento com $240^{\circ} \mathrm{C}$ verificou se maior perda de massa. Em relação a tensão máxima da dureza Janka no plano transversal, ocorreu um aumento significativo nas duas primeiras faixas de temperaturas $\left(160^{\circ} \mathrm{C}\right.$ e $\left.200^{\circ} \mathrm{C}\right)$, sendo recomendado o tratamento nas duas primeiras faixas de temperatura.

Em relação a análise colorimétrica, a utilização de tratamentos térmicos causou escurecimento da madeira, sendo a maior variação de cor observada no tratamento a $240^{\circ} \mathrm{C}$ para a madeira de Pinus elliottii. 
Tabela 2. Correlação de Pearson entre as variáveis físicas, mecânicas e colorimétricas analisadas no estudo.

\begin{tabular}{|c|c|c|c|c|c|c|c|c|c|c|c|c|}
\hline & $\rho_{12 \%}$ & $\sigma_{\text {Máx }} T$ & $\mathbf{L}^{*} \mathbf{T}$ & $\mathbf{L}^{*} \mathbf{R}$ & a*T & a* $R$ & $\mathbf{b}^{*} \mathbf{T}$ & $b^{*} \mathbf{R}$ & $C^{*} \mathbf{T}$ & $\mathbf{C}^{*} \mathbf{R}$ & $h^{\circ} T$ & $h^{\circ} R$ \\
\hline$\rho_{12 \%}$ & 1 & $-0,05$ & $0,48^{*}$ & $0,44^{*}$ & $-0,44^{*}$ & $-0,56^{*}$ & 0,26 & 0,02 & 0,16 & $-0,08$ & $0,55^{*}$ & $0,48 *$ \\
\hline$\sigma_{\text {Máx }} T$ & & 1 & $-0,23$ & $-0,18$ & $0,53^{*}$ & $0,50 *$ & 0,21 & 0,36 & 0,30 & $0,43 *$ & $-0,23$ & $-0,14$ \\
\hline $\mathrm{L}^{*} \mathrm{~T}$ & & & 1 & $0,97^{*}$ & $-0,56^{*}$ & $-0,58^{*}$ & $0,70 *$ & $0,56^{*}$ & $0,56^{*}$ & 0,42 & $0,97 *$ & $0,96 *$ \\
\hline$L^{*} R$ & & & & 1 & $-0,48^{*}$ & $-0,55^{*}$ & $0,73^{*}$ & $0,63 *$ & $0,61 *$ & $0,50 *$ & $0,94 *$ & $0,99 *$ \\
\hline$a * T$ & & & & & 1 & $0,91^{*}$ & 0,12 & 0,28 & 0,31 & $0,43^{*}$ & $-0,64 *$ & $-0,53^{*}$ \\
\hline$a * R$ & & & & & & 1 & $-0,02$ & 0,27 & $0,17^{*}$ & $0,43^{*}$ & $-0,66^{*}$ & $-0,61^{*}$ \\
\hline$b^{*} T$ & & & & & & & 1 & $0,83^{*}$ & $0,98^{*}$ & $0,76^{*}$ & $0,66^{*}$ & $0,70^{*}$ \\
\hline$b * R$ & & & & & & & & 1 & $0,86^{*}$ & $0,99 *$ & $0,46^{*}$ & $0,60 *$ \\
\hline$C^{*} \mathrm{~T}$ & & & & & & & & & 1 & $0,83^{*}$ & $0,51^{*}$ & $0,57 *$ \\
\hline$C^{*} \mathrm{R}$ & & & & & & & & & & 1 & 0,31 & $0,45^{*}$ \\
\hline$h^{\circ} \mathrm{T}$ & & & & & & & & & & & 1 & $0,94^{*}$ \\
\hline$h^{\circ} \mathrm{R}$ & & & & & & & & & & & & 1 \\
\hline
\end{tabular}

Em que: $\rho_{12 \%}=$ massa específica aparente a $12 \% ; \sigma_{\text {Máx }} T=$ tensão máxima na face transversal; $L^{*} T=$ claridade na face tangencial; $a^{*} T=$ coordenada vermelho-verde na face tangencial; $b^{*} T=$ coordenada azul-amarelo na face tangencial; $C^{*} T=$ saturação da cor na face tangencial; $h^{\circ} T=$ ângulo de tinta na face tangencial; $L^{*} R=$ claridade na face radial; $a^{*} R=$ coordenada vermelho-verde na face radial; $b^{*} R$ = coordenada azul-amarelo na face radial; $C^{*} \mathrm{R}=$ saturação da cor na face radial; $h^{\circ}$ ângulo de tinta na face radial; * = significativo, teste feito com $95 \%$ de confiança, onde mais próximo de 1 , diretamente proporcional, e mais próximo de -1 , inversamente proporcional.

\section{REFERÊNCIAS}

ASTM - AMERICAN SOCIETY FOR TESTING AND MATERIALS. D14394: standard test methods for small clear specimens of timber. Philadelphia, 2014.

ASTM - AMERICAN SOCIETY FOR TESTING AND MATERIALS. D2017: standard test methods of accelerated laboratory test of natural decay resistance of woods. Philadelphia, 2005.

ASTM - AMERICAN SOCIETY FOR TESTING AND MATERIALS. D2395-17: standard test methods for density and specific gravity (relative density) of wood and wood-based materials. Philadelphia, 2017.

ATES, S., AKYILDIZ, M.H., OZDEMIR, H. Effects of heat treatment on Calabrian pine (Pinus brutia Ten.) wood. Bioresources, Raleigh, v.4, n.3, p.1032-1043, 2009.

AYDEMIR, D. et al. Investigating changes in the chemical constituents and dimensional stability of heat-treated hornbeam and Uludag fir wood. BioResource, v.6, n.2, p.1308-1321, 2011.

BOONSTRA, M.J. et al. Strength properties of thermally modified softwoods and its relation to polymeric structural wood constituents. Annals of Forest Science, v.64, n.7, p.679-690, 2007.

CADEMARTORI, P.H.G. et al. Modification of static bending strength properties of Eucalyptus grandis heat-treated wood. Materials Research, v.15, n.6, p.922-927, 2012.

CADEMARTORI, P.H.G. et al. Thermal modification of Eucalyptus grandis wood: variation of colorimetric parameters. Maderas. Ciencia y tecnología, v.15, n.1, p.57-64, 2013.

CALONEGO F.W. et al. Physical and mechanical properties of thermally modified wood from $E$. grandis. European Journal of
Wood and Wood Products, v.70, n.4, p.453-460, 2012.

CHEN, Y. et al. The effect of heat treatment on the chemical and color change of black locust (Robiniapseudoacacia) wood flour. BioResources, v.7, n.1, p.1157-1170, 2012.

CONTE, B. et al. Propriedades físicas e colorimétricas da madeira termorretificada de Pinus elliottii var. elliottii. Scientia Forestalis, v.42, n.104, 2014.

ESTEVES, B. et al. Influence of steam heating on the properties of pine (Pinus pinaster) and eucalypt (Eucalyptus globulus) wood. Wood Science and Technology, v.41, p.193-207, 2007.

ESTEVES, B. et al. Heat-induced colour changes of pine (Pinus pinaster) and eucalyptus (Eucalyptus globulus) wood. Wood Science and Technology, v.42, n.5, p.369-384, 2008.

HILL, C.A.S. Wood Modification: chemical, thermal and other processes. Chichester: John Wiley \& Sons, 2006.

MODES, K.S. et al. Efeito da termorretificação nas propriedades mecânicas das madeiras de Pinus taeda e Eucalyptus grandis. Ciência Florestal, v.27, n.1, p.291-302, 2017.

MOHAREB, A. et al. Effect of heat treatment intensity on wood chemical composition and decay durability of Pinus patula. European Journal of Wood and Wood, v.70, p.519-524, 2012.

MOURA, L.F. et al. Efeitos da termorretificação na perda de massa e propriedades mecânicas de Eucalyptus grandis e Pinus caribaea var. hondurensis. Floresta, v.42, n.2, p.305-314, 2012.

MOURA, L.F.; BRITO, J.O. Efeito da termorretificação sobre as propriedades colorimétricas das madeiras de Eucalyptus grandis e Pinus caribaea var. hondurensis. Scientia Forestalis, v.39, n.89, p.069-076, 2011. 
NISHINO, Y. et al. Relations between the colorimetric values and densities of sapwood. Journal of Wood Science, v.46, n.4, p.267$272,2000$.

PINCELLI, S.M. et al. Effect of thermal rectification on colors of Eucalyptus saligna and Pinus caribaea woods. Maderas. Ciencia y tecnología, v.14, n.2, p.239-248, 2012.

PONCSAK, S. et al. Improvement of the heat treatment of Jack pine (Pinus banksiana) using Thermo Wood technology. European Journal of Wood and Wood Products, v.69, n.2, p.281286, 2011.

SANTOS, J.A. Mechanical behaviour of Eucalyptus wood modified by heat. Wood Science and Technology, v.34, p.39-43, 2000.

VIITANIEMI, P.; THERMOWOOD. Modified wood for improved performance. Proceedings of wood the ecological material the4th Euro-wood symposium. Stockholm, p.22-23, 1997.

ZANUNCIO, A.J.V. et al. Termorretificação e colorimetria da madeira de Eucalyptus grandis. Floresta e Ambiente, v.21, n.1, p.85-90, 2014.

Recebido em 22-07-2019

Aceito em 06-09-2019 